DOI: $10.17533 /$ udea.efyd.v33n1a03

URL DOI: http://doi.org/10.17533/udea.efyd.v33n1a03

\title{
EDUCACIÓN FÍSICA Y EDUCACIÓN DEL CUERPO EN EL URUGUAY: JESS T. HOPKINS (1912- 1922) ${ }^{1}$
}

\author{
EDUCAÇÃO FÍSICA E EDUCAÇÃO DO CORPO
} NO URUGUAI: JESS T. HOPKINS (1912- 1922)

\section{PHYSICAL EDUCATION AND CORPORAL EDUCATION IN URUGUAY: JESS T. HOPKINS (1912-1922)}

\author{
Paola Dogliotti Moro \\ Magister en Ense anza Universitaria por la Universidad de la Rep blica. \\ Profesora Departamento de Ense anza y Aprendizaje del Instituto de \\ Educaci $n$ de la Facultad de Humanidades y Ciencias de la Educaci $n$ \\ de la Universidad de la Rep blica (Uruguay). \\ paoladogliottimoro@gmail.com
}

Dogliotti, D. (2014). Educaci n f sica y educaci $n$ del cuerpo en el uruguay: jess t. Hopkins (1912- 1922). Educaci n F sica y Deporte, 33 (1), 31-50, Ene-Jul 2014

1 Este trabajo forma parte del proyecto de investigaci $\mathrm{n}$ "La formaci $\mathrm{n}$ de los secretarios y directores del Instituto $T$ cnico de la Federaci $n$ Sudamericana de ACJ (1922 - 1950) y su influencia en la gestaci $n$ de la EF en el Uruguay" financiado por el Instituto Universitario de la Asociaci n Cristiana de J venes (IUACJ) de Montevideo. Tambi n forma parte de la tesis de maestr a titulada: "Cuerpo y curriculum: discursividades en torno a la formaci $\mathrm{n}$ de docentes de educaci $\mathrm{n} f$ sica en Uruguay (1874-1948)" y se enmarca en la intersecci $\mathrm{n}$ de dos I neas de investigaci n: 1-"Pol ticas educativas, cuerpo y curr culum" (PECUC) del Dpto. de Educaci n F sica y Pr cticas Corporales del Instituto Superior 


\section{RESUMEN}

En este trabajo se analizan las principales discursividades en torno al cuerpo y la educaci $n f$ sica presentes en Jess T. Hopkins, maestro de educaci $\mathrm{n} f$ sica por la universidad de Springfield College de la Young Men Christian Asociation (YMCA) norteamericana, primer Director T cnico de la Comisi n Nacional de Educaci n F sica (CNEF) del Uruguay entre 1912 y 1920, y encargado del primer curso intensivo de formaci $\mathrm{n}$ de maestros de educaci $\mathrm{n} f$ sica. Entre los principales hallazgos se destaca la influencia de la YMCA en el proceso gestacional de la CNEF en el Uruguay. El profesor de educaci $n \mathrm{f}$ sica, en tanto ayudante del $m$ dico, desplegaba dos tipos de intervenci $n$, uno denominado normaci $n$, que apuntaba al cuerpo individual, $\mathrm{m}$ quina, a las disciplinas ejercidas sobre I en tanto an tomo-pol tica del cuerpo, y otro denominado normalizaci n, que tomaba al cuerpo en tanto especie y apuntaba al gobierno de la poblaci $n$ (Foucault, 1976). Jess Hopkins mostraba una interpretaci $n$ del evolucionismo darwiniano tendiente a la degenerescencia (Vigarello, 2005).

PALABRAS CLAVE: Cuerpo, educaci n f sica, Hopkins, Uruguay, 1912-1922

\section{RESUMO}

Neste trabalho analizam-se as principais discursividades sobre o corpo e a educaç o f sica, presentes em Jess $T$. Hopkins, professor de educaç o $\mathrm{f}$ sica pela universidade de Springfield College da Young Men Christian Asociation (YMCA) norteamericana, primeiro Diretor $\mathrm{T}$ cnico da Comiss o Nacional de Educaç o F sica ( CNEF) do Uruguai entre 1912 e 1920, e encarregado do primeiro curso intensivo de formaç o de professores de educaç o f sica. Entre as principais descobertas destaca-se a influ ncia da YMCA no processo gestacional da CNEF no Uruguai. O professor de educaç o $\mathrm{f}$ sica, enquanto ajudante do $\mathrm{m}$ dico, desempenhava dois tipos de intervenç $\mathrm{o}$, um denominado normaç $\mathrm{o}$, que apontava ao corpo individual, $\mathrm{m}$ quina, s disciplinas exercidas sobre ele enquanto an tomopol tica do corpo, e outro denominado normalizaç o, que tomava o corpo enquanto esp cie e apontava ao governo da populaç o (Foucault, 1976). Jess Hopkins mostrava uma interpretaç o do evolucionismo darwiniano tendente degeneresc ncia (Vigarello, 2005).

PALAVRAS-CHAVE: Corpo, educaç of sica, Hopkins, Uruguai, 1912-1922

de Educaci n F sica (ISEF) de la Universidad de la Rep blica (UdelaR) y 2- "Pol ticas educativas, curr culum y ense anza" (PECE) del Dpto. de Ense anza y Aprendizaje del Instituto de Educaci n de la Facultad de Humanidades y Ciencias de la Educaci n (FHCE) de la UdelaR. 


\section{ABSTRACT}

This paper analyzes the main corporal and physical education discourses present in Jess T. Hopkins, physical education professor at Springfield College of the YMCA of North America; first Technical Director of the National Commission of Physical Education (CNEF) of Uruguay between 1912 and 1920, and in charge of the first intensive physical training education professors. Among the main findings, it is evident the influence of the YMCA in the gestational process of the CNEF in Uruguay.

The physical education professor as a doctor assistant performed two kind of interventions: normaci $n$ and normalizaci $n$. The first one refers to individual body, machine; discipline applied on it as long as anatomopolitics of the body. The second one implies the body concept as specie and the population control (Foucault, 1976). Jess Hopkins showed a Darwin's evolution interpretation with a tendency of degeneration (Vigarello, 2005).

KEY WORDS: Physical education, corporal education, discourses, normaci $n$, normalizaci $n$ 


\section{INTRODUCCIÓN}

Este trabajo se propone un rastreo de los principales modos de significar a la educaci $\mathrm{n} f$ sica y al cuerpo en momentos de una fuerte institucionalizaci $\mathrm{n}$ de la educaci $\mathrm{n}$ f sica en el Uruguay (1912-1922), a trav s de los principales textos escritos por Jess T. Hopkins, misionero proveniente de la YMCA. Es preciso destacar que, si bien a continuaci $\mathrm{n}$ se presentar $\mathrm{n}$ los principales datos biogr ficos de Hopkins para dar cuenta del contexto de poca y sus modos de inserci $n$ y aceptaci $n$ en el Uruguay, el nfasis a lo largo del trabajo no estar puesto en el pensamiento de determinadas personalidades, ni en el desarrollo de datos hist ricos, sino, por el contrario, en el an lisis de discurso.

Desde nuestra perspectiva, el discurso no es producto de una determinada personalidad brillante y tiene efectos sobre los sujetos; este no es due o y se or del discurso sino que es interpelado por este, es puesto en las derivas de la cadena significante. En este sentido, se busca distanciarse del relato de los grandes hombres y se intenta poner el acento en las discursividades implicadas y manifestadas a trav s de las principales obras de Jess T. Hopkins ${ }^{2}$.

Jess T. Hopkins ${ }^{3}$ fue uno de los principales impulsores de la creaci $n$ de las plazas de deportes ${ }^{4}$ de la CNEF en su primera

2 Se busca percibir la singularidad de los sucesos, no se opone a la historia, sino al despliegue metahist rico de las significaciones ideales, a la b squeda del "origen" y de una esencia (Foucault, 1992, p. 7-11).

3 Naci en lowa, Estados Unidos, el 31 de marzo de 1887. Ingres en el a o 1906 al Internacional YMCA College de Springfield, Massachussets. Se recibi de Bachiller en Educaci n F sica (BPE) y posteriormente de Maestro en Educaci n F sica (MPE). Entre 1907 y 1912 desempe varios cargos de Director de Educaci n F sica de las ACJ, de las Escuelas $\mathrm{P}$ blicas de Moncton, en Canad, en la Asociaci $\mathrm{n}$ de Billings, de Montana, y en la de Gorgona, de Panam .

4 Las plazas de deportes se crearon en nuestro pa s a partir de la creaci $\mathrm{n}$ de la CNEF. Fueron construidas en base al proyecto de su primer presidente, Smith (1913), Ilamadas tambi n "plazas vecinales de cultura f sica" o "plazas de juego populares". Eran centros populares de 
d cada de existencia, miembro de la Comisi n Organizadora, como Secretario del Primer Curso Intensivo Te rico-Pr ctico para Maestros de Plazas de Deportes a inicios de $1920^{5}$. Fue Director de cuerpos de I deres de las diversas plazas de deportes y de la Asociaci n Cristiana de J venes. En 1912 se radic en Uruguay para encargarse del Departamento de Educaci n F sica de la Asociaci n Cristiana de J venes (ACJ), fundado en junio de $1912^{6}$. As fue valorado por sus correligionarios: introdujo en el

uso gratuito y ten an fines recreativos dirigidos a toda la poblaci $\mathrm{n}$. El modelo propuesto para su construcci $n$ proven a de Estados Unidos de Norteam rica. En 1920 la CNEF contaba con un total de 31 plazas de deportes y proyectaba la construcci n de 9 m s. En 1923 hab a 41 plazas instaladas y 27 proyectadas, de las cuales 7 se hallaban en construcci n (CNEF, 1923, p. 157). Para un an lisis del proyecto de Smith consultar Rodr guez (2012, pp. 181-187).

5 En el curso tuvo a cargo un tercio de las materias te ricas y algunas pr cticas: "Teor a y Pr ctica de la calistenia en el Gimnasio", "El Nuevo Concepto de la Educaci n F sica", "Pr ctica en el Gimnasio", "Por qu se ense a al ni o a jugar", "Qu es una Plaza de Deportes", "Principios Fundamentales de la Organizaci n de las Plazas", "Programas Modelos para Plazas de Deportes", "C mo organizar torneos, campeonatos, etc.", "Competencia en masa", "Construcci n y conservaci n de una Plaza de Deportes" (CNEF, 1920, pp. 1888-1890). En el Tercer Curso Intensivo Te rico-Pr ctico dict "Historia y Filosof a de la Educaci $n$ F sica" (CNEF, 1924, p. 10).

6 Felipe A. Conard, norteamericano, fundador en 1909 de la ACJ de Montevideo, relata el proceso conducente al viaje de Hopkins a Uruguay: "a los 18 meses de fundada la Asociaci n, el Sr. Ethan T. Colton, Secretario del Comit Internacional, nos visit y recomend que organiz ramos en forma el Departamento de Educaci n F sica, y luego colabor en la b squeda de un director" (Conard, 1959, p. 29). "Esta recomendaci n asombr a varios de nuestros amigos, como Don Eduardo Monteverde, quien nunca hab a pensado que la Asociaci n tuviera como uno de sus objetivos principales, la gimnasia y el deporte. Sin embargo, a medida que consultamos a amigos y funcionarios de las instituciones privadas y gubernamentales, encontramos una pronta recepci $\mathrm{n}$ de la idea; as es que pedimos al Comit que nos consiguiera los servicios de un director experimentado. La elecci $n$ no pudo haber sido mejor. El Sr. Jess T. Hopkins demostr poseer un magn fico car cter y una rica experiencia, 
pa s una orientaci n nueva del deporte, la instituci n del cuerpo de I deres, el examen fisico-m dico previo al ejercicio, el 'juego recreativo para todos' y destacando valores morales de la educaci $n$ f sica [...] En esos a os introdujo al pa s muchos juegos, como el b squet-ball, voley-ball, etc., que pronto se popularizaron (Uruguay, 1929, pp. VII-VIII).

En 1913 fue designado Director General de Plazas Vecinales y luego Director T cnico de la CNEF, desde donde impuls el establecimiento de plazas de deporte. Entre los a os 1917 y 1918 regres a su pa s para escribir su tesis de graduaci n, "A monograph on physical education written in Spanish". En 1919 retorn a Montevideo y ocup el cargo de Director Continental de Educaci n F sica de la Federaci n Sudamericana de ACJ, con sede en esta ciudad. Desde su regreso ocup el cargo de Asesor T cnico Honorario de la $\mathrm{CNEF}^{7}$ hasta que en 1928 residi en forma definitiva en Estados Unidos. Fue representante t cnico en Am rica del Sur del Comit Ol mpico Internacional (Uruguay, 1929 pp. VII-IX; CNEF, 1920, 1924; Pereyra, 1946).

Entre otras de sus producciones se destaca la organizaci $n$ del libro Reglamentos Oficiales de Juegos Atl ticos y Estudios sobre educaci $n$ f sica moderna (Uruguay, 1929), en el que se detalla:

La publicaci $n$ de este libro se debe a la iniciativa del Sr. Jess T. Hopkins, Secretario de la Secci n Educaci n F sica de la Federaci n Sudamericana de Asociaciones Cristianas de J venes. Fue I quien hizo las gestiones para que la conocida Editorial "Spalding" publicara la primera edici $\mathrm{n}$ : quien recolect la mayor parte del material, estudi las mejoras a introducirse en aquella. [...] Fue en-

no solamente en Educaci $\mathrm{n} F$ sica, sino que tambi $\mathrm{n}$ en el programa total de la Asociaci n" (Conard, 1959, p. 33). "Y cuando lleg el Director, Jess T. Hopkins, no tard en demostrar lo oportuno del proyecto, pues el registro social de 200 socios se aument con 400 nuevos, y pronto el hombre se relacion con la Universidad y con la Comisi $n$ Nacional de Educaci n F sica" (Conard, 1959, p. 29).

7 Por resoluci n № 5.065, del 3 de mayo de 1919 (Blanco, 1948, p. 336). 
tonces que, en atenci $\mathrm{n}$ a que I era, en cierto sentido, el verdadero autor del libro, y en reconocimiento de su larga y fruct fera actuaci $\mathrm{n}$ en el desarrollo de la educaci $\mathrm{n} f$ sica en Sudam rica, y a insinuaci $n$ de sus colaboradores en la redacci $n$ de aquel, se resolvi dedicarle su propio libro, Itimo trabajo que realiz en estos pa ses (Uruguay, 1929, p. V).

Las fuentes seleccionadas y analizadas en la presente investigaci $\mathrm{n}$ son:

1) La tesis de graduaci $n$ mencionada. Difundida en Uruguay, la escribi en espa ol y muchos de los textos citados fueron publicados en la Revista Uruguay-Sport, publicaci n oficial de la CNEF. Ra I V. Blanco ${ }^{8}$, refiri ndose a ella en el primer libro sobre recreaci n escrito por un uruguayo, expresa que Hopkins realiz una autorizada investigaci n sobre los juegos y su origen y "marc la era del oro de los deportes y la educaci $n \mathrm{f}$ sica en Uruguay, siendo las Plazas de Deportes su obra m s importante organizada" (Blanco, 1940, p. 22).

2) El art culo titulado "Educaci n F sica. Bases Biol gicas de los juegos atl ticos" publicado en 1922 en la Revista Uruguay Sport, de la CNEF.

8 Egresado de la Escuela de Leaders de la YMCA de Montevideo, a partir de ello pas directamente a la docencia. Fue Director de la Plaza de Deportes № 5 de la CNEF. Visit en gira varios pa ses de Europa y Am rica. En 1938, concibi la necesidad de organizar el Primer Congreso Nacional de Educaci n F sica, como base para celebrar el primero de car cter panamericano. Previo a la necesidad de establecer los Congresos Panamericanos, present en 1941 la idea de creaci n del Instituto Panamericano de Educaci n F sica y estructur su fundaci $n$ con sede en Estados Unidos. Fue Asesor T cnico del Comit Nacional de Deportes y Asesor T cnico el Comit Ol mpico del Per . Entre sus libros se destacan: Educaci n F sica. Un panorama de su historia y Recreaci n. JuegosDanzas-Rondas Musicales-Luchas. Su historia y fundamentos. Su aspecto fisiol gico y pedag gico. Su valor moral y $f$ sico, libro que mereci el Gran Diploma de Honor otorgado en Cuba (Blanco, 1948 y 1940). 
Para dar cuenta, m s a n, de la gran influencia ejercida por la YMCA norteamericana en el proceso gestacional de la CNEF y de la educaci $\mathrm{n}$ f sica en Uruguay, y sobre todo de las vinculaciones entre la conducci n pol tica del pa s y la ACJ, transcribimos algunos $\mathrm{p}$ rrafos de la carta enviada por Jess T. Hopkins a J.H. McCurdy, Director de Springfield College, el 26 de agosto de 1912, a solo un poco $\mathrm{m}$ s de dos meses de su arribo a Montevideo:

Conard, nuestro secretario y un graduado de la Universidad de Illinois, y Monteverde, su asociado, un uruguayo muy influyente, han sentado las bases para un maravilloso trabajo de la asociaci $n$ en este peque o pero importante pa s. [...] En poco $\mathrm{m} s$ de tres a os, estos dos hombres han construido una membres a de 750 de los cuales 6/7 son uruguayos; tienen en su comisi $n$ algunos de los hombres $\mathrm{m}$ s fuertes de la ciudad; han asegurado el activo inter s de la mayor a de los hombres influyentes en el cuerpo diplo$\mathrm{m}$ tico. Monteverde pas una hora con el presidente ${ }^{9}$ hace poco tiempo describi ndole nuestro trabajo y el Presidente odia todo lo que sabe a cristianismo, pero cuando termin se fue con el ejecutivo alabando nuestro trabajo. Este Presidente ha designado una Comisi n Nacional de Educaci n F sica y el Congreso ha destinado $\$ 50.000$ por a o para su trabajo. El Presidente de la Asociaci n, Mr. Towers, es miembro de esta Comisi $n, y$ ha aceptado la designaci $\mathrm{n}$ con el solo prop sito de usar su influencia para poner al Director $\mathrm{F}$ sico de la Asociaci $\mathrm{n}$ en buenas relaciones con esta Comisi n. Y esto, me complace decirlo, est funcionando muy bien. La Comisi n est planificando un sistema de plazas de deportes y justo esta tarde el presidente ${ }^{10}$ vino en su autom vil para llevarme

9 Jos Batlle y Ord ez. Las vinculaciones de Batlle con la ACJ son importantes; entre otras, fue compa ero de escuela ("Escuela Adams") de Pedro C. Towers, quien fue el Presidente fundador de la ACJ de Montevideo, contador en la creaci n del Banco de la Rep blica, luego Gerente del Banco Espa ol del R o de la Plata. El Presidente Batlle le ofreci la gerencia del Banco Rep blica pero ya hab a aceptado el puesto en El Espa ol (Conard, 1959, p. 28).

10 Juan A. Smith. 
a inspeccionar el sitio de la primera plaza. Me dio un bosquejo de sus planes y me pidi para reunirse conmigo ma ana y ofrecerle las sugerencias y $\mathrm{cr}$ ticas que desee.

Agosto, 29: Tuve que interrumpir esta carta hasta ahora. Tuve el placer de pasar una hora y media con el Presidente y Tesorero de la CNEF ayer discutiendo los planes para sus plazas. Debo entregar un plan y detallar una lista de equipamiento ma ana para su aprobaci $n$. Adem s sugirieron que les gustar a que yo tuviera alg $\mathrm{n}$ tipo de relaci $\mathrm{n}$ oficial para la supervisi n. [...] Estamos muy entusiasmados con el asunto porque pr cticamente significa que podremos controlar la situaci $n$ de las plazas y si hacemos una buena impresi $n$ en la Comisi n, no hay duda de que podremos tener una gran influencia en todo el trabajo de este rgano nacional en sus esfuerzos por establecer un movimiento nacional por la educaci $\mathrm{n} f$ sica.

El Inspector Nacional de Escuelas $\mathrm{P}$ blicas ${ }^{11}$ ha pedido una entrevista con el prop sito de hablar sobre la educaci $\mathrm{n} f$ sica en las escuelas $p$ blicas. Sus escuelas $p$ blicas est $n$ pobremente ubicadas actualmente en varios cientos de peque as casas y salones, etc. esparcidos por la ciudad. Dan pena en cuanto a la sanidad e higiene. Toda la EF que se haga debe ser en el sal n. Los patios son desconocidos, y ni hablar de gimnasios.

Visitamos todos los gimnasios de la ciudad poco despu s de nuestra llegada y encontramos que muchos de ellos est $\mathrm{n}$ en la "Dark Ages". Equipados con un ring de boxeo en el centro y despu s numerosas pesas, etc. esparcidas alrededor. Est $n$ administrados por el t pico profesor hombre fuerte. Las paralelas y caballos que tienen son hechos artesanalmente, y fueron importados de Europa hace cuarenta a os. Supongo que le costar creer que el gimnasio que la Asociaci $\mathrm{n}$ est acondicionando -una vieja iglesia- ser el segundo mejor gimnasio en Sudam rica -el primero es el nuevo edificio de Bs. As. Por supuesto que no he estado en la costa oeste, pero R o, Bs. As. y Montevideo son las tres ciudades $\mathrm{m} \mathrm{s}$ modernas en el rea de gimnasios por aqu. Salassa, en $\mathrm{R}$ o

11 Abel $P$ rez. 
y Phillips, en Bs. As. encontraron exactamente las mismas condiciones que yo estoy encontrando aqu. Nuestros aparatos, clases masivas con m sica, y muchos juegos asombran a todos. Luego, cuando hablamos de sanidad, higiene personal y trabajo de salud municipal quedan completamente shockeados.

Tomando todo esto en consideraci n: la CNEF, las Escuelas P blicas, la Universidad, y el Sistema de Plazas de Deportes; en ning $\mathrm{n}$ lado encontrar un departamento $\mathrm{f}$ sico de la Asociaci $\mathrm{n}$ una situaci $\mathrm{nm}$ s promisoria y nica para un maravilloso trabajo de extensi $\mathrm{n}$. Estoy muy feliz de tener esta oportunidad (Hopkins, 1912) ${ }^{12}$.

La carta da cuenta de la visi n de un extranjero proveniente del "primer mundo" y su lectura de la realidad uruguaya y de esta regi $n$, y el impacto que ocasion la venida de los "misioneros" a Sudam rica. A continuaci n nos abocaremos al an lisis espec fico de los modos de entender al cuerpo y a la educaci $\mathrm{n} f$ sica a lo largo de sus textos.

\section{RESULTADOS \\ Evolucionismo y eugenismo: degenerescencia}

Para explicar las bases biol gicas de los juegos at ticos, Hopkins se basa en fundamentos de un evolucionismo darwinista:

Se ven en el cuerpo humano las huellas de evoluci $\mathrm{n}$ de la raza a trav s de las edades, como Darwin nos las hizo ver. [...] El ser humano durante el per odo de su crecimiento recoge los beneficios de la evoluci n de la raza (Hopkins, 1922, pp. 3.494-3.495) ${ }^{13}$.

12 Carta traducida del ingl s por la licenciada Mercedes Couchet.

13 'La 'Se orita Naturaleza' es una maestra y gu a infalible si la dejamos actuar libremente, porque as como el embri $n$ recapitula biol gicamente la evoluci $\mathrm{n}$ de la raza, as tambi $\mathrm{n}$ el ni o recapitula etnol gicamente las generaciones por las que el ser humano ha pasado" (Hopkins, 1918, p. 19). 
Sin embargo, estos argumentos de progreso y mejoramiento de la raza tergiversan la teor a darwiniana y constituyen, seg n Vigarello (2005), una teor a de la degenerescencia: la inspiraci $n$ extra da de Darwin, por ejemplo, no deja de traicionar la argumentaci $n$ seleccionista al postularle una finalidad que no tiene. La selecci $\mathrm{n}$ natural no debe ser interpretada desde el ngulo normativo de un "mejoramiento" gradual de ciertas especies o lo contrario con respecto a otras: "Lo que garantiza la selecci $n$ natural darwiniana no se encuentra de ninguna manera marcado por el sello de la ideolog a del progreso. Una evoluci $n$ por adaptaci $n$ ecol gica no prejuzga en absoluto el perfeccionamiento de la raza" (Conry, p. 329). Las fuerzas que seleccionan las especies y las diferencian "transforman", pero no "degradan" ni "mejoran". El propio Darwin hab a respondido a los enfoques que se esforzaban por ver "avances" o "retrocesos", normalizando una evoluci n o antievoluci n. La teor a de la "degenerescencia" es normativa, al contrario del evolucionismo darwiniano (Vigarello, 2005, p. 111).

Para dar estos fundamentos recurre a doctores de su pa s $(\mathrm{Gu}-$ lick, G. Stanley Hall, McCurdy, McKenzie y Fisher) que "han estudiado al ser humano a trav s de la historia de la raza" (Hopkins, 1922, p. 3.494). Seg n estas ideas, el ni o pasa en sus etapas de crecimiento por las mismas etapas de la historia de la humanidad. En esta teor a el cuerpo org nico es el motor del desarrollo mental:

Por los grandes adelantos filos ficos, basados en la teor a de la evoluci n, ahora sabemos que los ni os griegos, que dedicaban tantas horas diarias a los deportes ${ }^{14}$, no desarrollaban, nicamente, sus cuerpos sino que tambi n cultivaban sus intelectos. El sistema muscular es la llave del desarrollo del sistema nervioso (el cerebro, etc.) y los rganos vitales (Hopkins, 1918, p. 9).

14 Hopkins idealiza la cultura griega y denomina a sus pr cticas corporales "deportes", desde una visi n etnocentrista moderna. 
Aqu sientan las bases de un plan de educaci $\mathrm{n} f$ sica "basado en un estudio del desarrollo de la raza" (Hopkins, 1918, p. 9). Las bases biol gicas son el suelo epist mico de esta disciplina: "dice el Doctor Tyler, que nuestro sistema muscular est estratificado. Es decir, que hemos adquirido diversas partes en distintas pocas de la evoluci n" (Hopkins, 1918, p. 12).

Por tal motivo, "toda educaci n, y especialmente la educaci $\mathrm{n} f$ sica debe estar basada en un estudio biol gico y etnoI gico de la raza humana" (Hopkins, 1918, p. 43). Para ello lo central no es qu sistema de educaci $n$ f sica elegir sino que est de acuerdo con el "desarrollo natural". Lo org nico y las etapas del desarrollo son sin nimo de "natural". No existe para el positivismo evolucionista una distancia entre el objeto real y el objeto de conocimiento, sino que los desarrollos cient ficos de las ciencias biol gicas son presentados como "la naturaleza" a respetar, y todos los presupuestos que se desprenden de "la ciencia" son los que determinan los modos de regulaci $\mathrm{n}$ de la vida org nica del hombre, en este caso, mediante ejercicios $f$ sicos. "La cuesti $n$ es que cualquier procedimiento, para ser bueno o aceptable, debe tener alguna base cient fica que no viole ninguna ley del crecimiento natural. Debe ayudarlo y no impedirlo" (Hopkins, 1918, p. 43).

La ciencia positiva se erige como la verdad incuestionable y no se percibe que sus "descubrimientos" son producto de una episteme y de un corpus te rico desde donde se mira el objeto y se lo construye. Se olvida que el cuerpo org nico, an tomo-fisiol gico y evolutivo no es un estado de naturaleza (si es que pudiera haber una instancia tal) sino producto de una serie de dispositivos construidos discursivamente. Seg n Hopkins, siguiendo los dict menes de las etapas evolutivas del desarrollo, cualquier sistema de educaci $\mathrm{n} f$ sica puede ser utilizado, aunque "teniendo en cuenta la preferencia personal o el temperamento nacional. [...] Supongo que para la psicolog a 
sueca no hay mejor sistema que el de Ling, as como para los alemanes el que origin Jahn" (Hopkins, 1918, p. 43).

Lo tnico adquiere relevancia en el evolucionismo, lo gen tico determina el comportamiento social del ni o, de una raza, de un pa s. "Estos instintos ${ }^{15}$ que hemos heredado pueden ponerse en pr ctica en forma de juego" (Hopkins, 1918, p. 22); "casi todas las manifestaciones sociales del chico son reverbaraciones o reflejos de sus antepasados" (Hopkins, 1918, p. 20). Hopkins trata los movimientos construidos en el intercambio del hombre con la cultura como si fueran instintos. "El muchacho es un verdadero indio incivilizado o peque o salvaje. [...] Representa muy bien al hombre de poca civilizaci n" (Hopkins, 1918, p. 27). Se equipara civilizaci $n$ a educaci $n$ y cultura, e incivilizaci $n$ a salvajismo y falta de cultura.

As, se establecen "razas superiores", las primeras, e "inferiores", las segundas: en palabras de McKenzie, Hopkins expresa que "por el cultivo de las grandes acciones fundamentales y vitales es que el hombre civilizado ha mantenido su superioridad sobre las razas primitivas y un programa de educaci $\mathrm{n}$ f sica debe estar basado en ensayos de esas acciones" (Hopkins, 1918, p. 63). La educaci $\mathrm{n}$ f sica contribuye, de este modo, a construir una raza "superior", "civilizada", "m s fuerte", y a combatir los males de su "degeneraci $n$ ". As,

Nosotros somos [...] los culpables del crimen de ignorar el debido funcionamiento de este organismo y de haberlo dejado degenerar. [...] Notaremos [...] s ntomas de toda clase de enfermedades $\mathrm{f}$ sicas y morales [...] debido a su ignorancia o a su descuido, cada $\mathrm{d}$ a avanzan por un camino que los conduce hacia un estado $f$ sico, moral y mental, m s degenerado" (Hopkins, 1918, p. 4).

15 Por ejemplo: el instinto de nado, "cuyo origen fue el tiempo en que nuestros antepasados viv an cerca de las aguas" (Hopkins, 1918, p. 21). 


\section{La educación física, entre la anatomía política del cuerpo y la biopolítica}

En el discurso evolucionista de la degeneraci $n$, la educaci $n$ $\mathrm{f}$ sica es presentada como una herramienta esencial para combatirla, una tecnolog a de poder. En este sentido,

...el viejo derecho soberano de poder matar necesita ahora ser justificado y ejercido biol gicamente. Ya no es la supresi n del enemigo pol tico lo que est en juego en esta nueva fisiolog a del poder de muerte del Estado, sino la eliminaci $\mathrm{n}$ de la amenaza biol gica, de la degradaci n, de lo anormal (Castro, 2011, p. 58).

Para dar este combate, se ala Hopkins que "en Nueva York existe una instituci n llamada 'The Life Extension Institute'. Su t tulo indica que es un instituto para ayudar a prolongar la vida. Est dirigido por un cuerpo de expertos en todos los detalles del organismo humano" (Hopkins, 1918, p. 6). Un cuerpo de m dicos se encarga de realizar ex menes y controles de todos los rganos y su funcionamiento:

Hacen una inspecci $\mathrm{n}$ absoluta. Adem s [...] hacen preguntas que revelan los $\mathrm{h}$ bitos higi nicos del individuo: qu come, cu nto tiempo duerme, qu clases de ejercicios $\mathrm{f}$ sicos practica; $\mathrm{cu}$ ntos cigarros fuma; cu nto caf toma; si toma bebidas alcoh licas; si est constipado, etc. Despu s de estudiar los resultados del examen le env an una carta en la cual le aconsejan y le recomiendan la manera $\mathrm{m} \mathrm{s}$ conveniente de vivir. [...] Esta instituci $\mathrm{n}$ est revisando miles de personas cada a o (Hopkins, 1918, p. 6).

Se realiza un control infinitesimal sobre cada uno de los cuerpos: h bitos de vida, costumbres, pero tambi $\mathrm{n}$ el saber $\mathrm{m}$ dico tiene el poder sobre la vida de la especie humana:

Compa as de seguros sobre la vida pagan a esta Instituci n para que revise a sus asegurados. Si pueden prolongar la vida de sus 
asegurados es un buen negocio para la Compa a! Tambi n una $f$ brica que emplea miles de obreros considera dinero bien gastado el pagar por la revisi n completa de sus empleados, pues con el mejoramiento de la salud de ellos, resultar $\mathrm{n}$ trabajadores $\mathrm{m} \mathrm{s}$ eficientes (Hopkins, 1918, p. 7).

La anatom a pol tica del cuerpo y la biopol tica ejercida por la medicina tienen su $\mathrm{m}$ xima expresi $\mathrm{n}$ en un pa s con un capitalismo econ mico prominente. El profesor de educaci $\mathrm{n} f$ sica se convierte aqu en un aliado fundamental del $\mathrm{m}$ dico,

A nosotros nos corresponde hacer lo que est a nuestro alcance para complementar la labor de la ciencia. Debemos de ense ar a los chicos a vivir como es debido y as obtener salud, fuerza y eficiencia para los componentes del organismo (Hopkins, 1918, pp. 7-8).

\section{Muscularización de la moral: los músculos como "órganos de la voluntad"}

Para Hopkins, el cuerpo es el de la an tomo-fisiolog a: "no es demasiado esperar que el hombre sepa tanto del mecanismo y operaciones de su organismo, como sabe de su jautom vil! o de su ¡caballo!" (Hopkins, 1918, p. 17) ${ }^{16}$. Mediante la educaci n f sica, "cuando llegue a ser un hombre poseer rganos vitales sanos, fuertes y llenos de vigor, y una musculatura suficiente para dar al cuerpo esbeltez, buena postura y agilidad al intentar acciones que requieran coordinaci n, etc." (Hopkins, 1918, p. 17). Estos atributos dan cuenta de una construcci $n$ masculina de los cuerpos. De este modo concibe a los m sculos

16 Si bien "hombre" era la palabra de uso corriente en el momento para referirse en modo gen rico a ambos g neros, a trav $\mathrm{s}$ de esta met fora queda claro que se est refiriendo al g nero masculino; prueba de ello es que Hopkins dedica un apartado de su tesis a la "educaci $\mathrm{n} f$ sica de la mujer". 
como " rganos de la voluntad" y al "car cter como una red de h bitos neuro-musculares" (Hopkins, 1922, p. 3.494). Todo est biologizado para Hopkins, hasta la personalidad; la constituci $n$ subjetiva del ser humano no es tal sino que est determinada por los m sculos:

Los $\mathrm{m}$ sculos son los que generan el h bito, la imitaci $\mathrm{n}$, la obediencia, el car cter y casi todas las maneras y costumbres. Se puede decir que la resistencia, la destreza y la perseverancia son virtudes musculares y que la fatiga, el capricho, la irascibilidad, la falta de control y el desequilibrio son deficiencias musculares (Hopkins, 1922, p. 3.494).

Podr amos llamar a esto una biolog a moralizada y una moral biologizada; ambos aspectos est $\mathrm{n}$ presentes: por un lado, al $\mathrm{m}$ sculo se le atribuyen aspectos de orden moral y por otro, las cualidades morales son musculares.

\section{CONSIDERACIONES FINALES}

En relaci $\mathrm{n}$ a c mo se entiende el cuerpo, lo que da cuenta de los modos de constituci $\mathrm{n}$ epist mica de la educaci $\mathrm{n} f$ sica, se alamos que lo predominante es el cuerpo org nico, en tanto materia viviente. Pero, en este sentido, seg $\mathrm{n}$ la distinci $\mathrm{n}$ griega, m s cercano a la zoe que a la $b$ os.

Si tenemos en cuenta la distinci $n$ griega entre b os y zo $\quad[\ldots]$ no deber amos hablar de biopol tica, sino de zoopol tica. Este ser a el caso cuando el objeto de la biopol tica no es la vida humana en su sentido espec fico, sino la vida biol gica de los hombres. Que el t rmino "bios" se haya impuesto sobre "zoe", que lo haya incluso sustituido y que, por ello, hablemos de "biopol tica" y no de "zoopol tica", se debe a la aparici n a comienzos del siglo XIX del t rmino "biolog a", cuya invenci $n$ se atribuye [...] al franc $s$ Jean-Baptiste Lamarck (1744-1837) (Castro, 2011, p. 19). 
La educaci $\mathrm{n} f$ sica se despliega como una de aquellas instancias que prolongan la vida. De este modo, a trav s del desarrollo de las ciencias $\mathrm{m}$ dicas, la educaci $\mathrm{n} \mathrm{f}$ sica tiene un papel fundamentalmente higi nico, en tanto act a sobre la salud y en el mbito de la prevenci $\mathrm{n} \mathrm{m}$ s que en el de la enfermedad. El profesor se convierte en un ayudante del $\mathrm{m}$ dico y, para esto, recibe de este campo una serie de conocimientos terap uticos e higi nicos y diferentes $t$ cnicas para desarrollar una ortopedia sobre el cuerpo. A partir de aqu se despliega, con mayor o menor grado, una infinidad de $\mathrm{t}$ cnicas de medici $\mathrm{n}$ y clasificaci $\mathrm{n}$ de los cuerpos a trav $\mathrm{s}$ de los test posturales y de aptitud f sica. Desde los diversos diagn sticos, test, pruebas, ex menes y estad sticas elaboradas a partir de sus resultados, se desplegaban dos tipos de intervenci n: uno, denominado normaci $n$, que apuntaba al cuerpo individual, $m$ quina, a las disciplinas ejercidas sobre I en tanto an tomo-pol tica del cuerpo, y otro denominado normalizaci $n$, que tomaba al cuerpo en tanto especie y apuntaba al gobierno de la poblaci n (Foucault, 1992). Pero la vida escapa al sue o clasificador; siempre queda un resto que excede a ambos mecanismos de normaci $n$ y normalizaci $n$.

En la Modernidad, a la vida biol gica se la produce y se la administra. Podr a decirse incluso que -en la Modernidad, insistimos- la vida es objeto de biologizaci n, de normalizaci $n$ bioI gica. Pero ella es tambi n, como veremos, lo que nunca queda exhaustivamente atrapado en los mecanismos que pretenden controlarla; siempre los excede y se les escapa. Las categor as de gobierno y de gubernamentalidad, hacia las que se encamina el an lisis foucaulteano del biopoder, buscan precisamente dar cuenta de lo uno y de lo otro (Castro, 2011, p. 39).

Jess Hopkins, a trav s de su escritos, mostraba una interpretaci $\mathrm{n}$ del evolucionismo darwiniano tendiente a la degenerescencia (Vigarello, 2005), lo que implicaba una tergiversaci $n$ de la teor a de Darwin, en tanto la evoluci n era vista como un proceso de antievoluci $\mathrm{n}$ normativa, como un mecanismo de

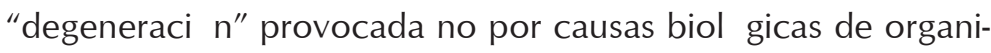


zaci n interna de los cuerpos, sino como consecuencia de los fen menos sociales del industrialismo.

En esta discursividad, el cuerpo era principalmente un "conjunto de m sculos", y estos se conceb an como " rganos del cerebro", "de la voluntad, del pensamiento y del sentimiento", mientras el car cter se entend a como un "plexo de h bitos motores". En cierta medida se presenta una biologizaci $n$ de la moral o, m s que esto, una muscularizaci $n$ de la moral.

Si se comparan las discursividades presentes en los diversos documentos escritos por Hopkins con las de otras personalidades que tuvieron un rol importante en la configuraci $\mathrm{n}$ de la educaci $\mathrm{n}$ del cuerpo en el Uruguay del Novecientos -Jos Pedro Varela, los presentes en el Congreso Pedag gico de 1882, Alejandro Lamas, Jos Batlle y Ord nez, la C mara de Fomento que justific el Proyecto de Ley de creaci $n$ de la CNEF, las $p$ ginas de la revista Uruguay Sport, Julio J. Rodr guez ${ }^{17}$-, podemos decir que en todas ellas, con diferentes modalidades, se presenta un eugenismo pronunciado; la gimn stica, la cultura $f$ sica, la educaci $n$ f sica o los sports son herramientas indispensables para el "mejoramiento de la raza". Se idealizan o ponen en un lugar de jerarqu a a las razas anglosajonas frente a las latinas, mostrando ciertos rasgos de racismo. Se presenta a las primeras como "superiores", "civilizadas" y "educadas", y a las segundas con "falta de cultura", "incivilizadas" y con diversos grados de "salvajismo" o "barbarie".

Desde aqu se prescriben y delinean determinados tipos de cuerpos y se relegan otros, muchas veces asociados con la enfermedad y la pobreza. Si bien se habla en el gen rico de "hombre" se est representando al cuerpo del var n; se idealiza el cuerpo del "gentleman" ingl s, vigoroso, esbelto, recto, $r$ gido, con altivez de postura, tonalidad en su rostro, fuerte.

A trav s de la figura de Jess T. Hopkins, una de las discursividades que mayor influencia tuvo en la matriz fundacional que

17 Para un an lisis de todas ellas consultar Dogliotti (2012). 
constituy el proceso de creaci $n$ de la CNEF fue la proveniente de la YMCA de Estados Unidos. A partir del proceso gestacional de la CNEF, hay un cambio importante en cuanto a que la incidencia que comienza a ejercer Estados Unidos para la conformaci $\mathrm{n}$ del campo de la educaci $\mathrm{n} f$ sica en Uruguay es mayor que la europea. Espec ficamente, la influencia de la YMCA se realiza a trav s de la llegada a Latinoam rica de egresados de sus universidades, formados en educaci $\mathrm{n} f$ sica. Pero la influencia ejercida en Uruguay es mayor que en otros pa ses del continente. A diferencia, por ejemplo, de Argentina, cuando llegan los norteamericanos a Montevideo el desarrollo de la educaci $n \mathrm{f}$ sica era $\mathrm{m} \mathrm{s}$ que precario y escaso.

En Argentina, a trav s de la figura de Romero Brest, ya hab a un desarrollo mayor de la educaci $\mathrm{n} f$ sica y el instituto de formaci $\mathrm{n}$ de profesores ya se hab a creado. En Uruguay coincide la llegada de Hopkins con la necesidad de desarrollo de toda la "cultura f sica" del pa s, reci n fundada la CNEF. Este hecho fue de trascendencia y llev , en cierta medida, a que en Uruguay, un pa s peque$\mathrm{o}$, la influencia de la YMCA fuera tan importante en estas $d$ cadas.

\section{REFERENCIAS}

1. Blanco, R. V. (1940). Recreaci n. Juegos-Danzas-Rondas Musicales-Luchas. Su historia y fundamentos. Su aspecto fisiol gico y pedag gico. Su valor moral y $f$ sico. Montevideo: Ediciones Indoam rica.

2. Blanco, R. V. (1948). Educaci n F sica. Un panorama de su historia. Montevideo: Impresora Adroher.

3. Castro, E. (2011). Lecturas foucaulteanas. Una historia conceptual de la biopol tica. La Plata: UNIPE, Editorial Universitaria.

4. CNEF Comisi n Nacional de Educaci n F sica (1918a). Uruguay-Sport. Archivos de la Comisi n Nacional de Educaci n F sica, 2.

5. CNEF Comisi n Nacional de Educaci n F sica (1918b). Uruguay-Sport. Archivos de la Comisi n Nacional de Educaci n F sica, 6.

6. CNEF Comisi n Nacional de Educaci n F sica (1920). Curso Intensivo te rico pr ctico para maestros de Plazas de Deportes. Uruguay-Sport. Archivos de la Comisi n Nacional de Educaci n F sica, 28, 1879-1890. 
7. CNEF Comisi n Nacional de Educaci n F sica (1923). Plan de Acci $n$ de la CNEF y conclusiones que se derivan del mismo. Presentado por su Director T cnico Sr. Julio J. Rodr guez. Montevideo: CNEF.

8. CNEF Comisi n Nacional de Educaci n F sica (1924). Informes, horarios y otros detalles del Tercer Curso Intensivo Te rico-Pr ctico para la preparaci $n$ de maestros de educaci $n$ f sica a realizarse en Piri polis del 17 al 29 de Marzo de 1924. Montevideo: CNEF.

9. Conard, F. (1959). Memorias de la Fundaci $n$ de la Asociaci $n$ Cristiana de J venes de Montevideo. Montevideo: Asociaci n Cristiana de J venes de Montevideo.

10. Conry, Y. (1974). L'introduction du darwinisme en France au XIX si cle. Paris: Urin.

11. Dogliotti, P. (2012). Cuerpo y curriculum: discursividades en torno a la formaci $n$ de docentes de educaci n f sica en Uruguay (1874-1948) (Tesis de Maestr a). Montevideo: FHCE, Universidad de la Rep blica.

12. Foucault, M. (1976). Historia de la sexualidad. Tomo I. La voluntad de saber. Madrid: Siglo XXI.

13. Foucault, M. (1992). Nietzsche, la genealog a, la historia. En Genealog a del poder (pp. 7-29). Madrid: La Piqueta.

14. Hopkins, J. T. (1912). Carta a J. H. McCurdy, 26 de agosto de 1912. Montevideo: CNEF.

15. Hopkins, J. T. (1918). A monograph on physical education written in Spanish (Graduation Thesis). Springfield, Massachusetts: International Young Men's Christian Association College.

16. Hopkins, J. T. (1922). Educaci n F sica. Bases biol gicas de los juegos atl ticos. Uruguay-Sport. Archivos de la Comisi n Nacional de Educaci n F sica, 52, 3493-3498.

17. Pereyra, J. (1946). Semblanza del Sr. Jess T. Hopkins. Montevideo: Centro Cultural de Profesores de Educaci $n$ F sica.

18. Rodr guez, R. (2012). Saber del cuerpo: una exploraci n entre normalismo y universidad en ocasi $\mathrm{n}$ de la educaci $\mathrm{n}$ f sica (Uruguay, 1876-1939) (Tesis de Maestr a). Montevideo: FHCE, Universidad de la Rep blica.

19. Smith, J. A. (1913). Plazas vecinales de cultura f sica. Montevideo: Barreiro y Ramos.

20. Uruguay (1929). Reglamentos oficiales de juegos atl ticos y estudios sobre educaci n f sica moderna. Buenos Aires-Montevideo: Mundo Nuevo.

21. Vigarello, G. (2005). Corregir el cuerpo. Historia de un poder pedag gico. Buenos Aires: Nueva Visi n.

Recepción: 05-10-2013

Aprobación: 05-03-2014 